\title{
Effect of adrenalectomy and glycemic status on caloric efficiency and adiposity in the congenic LA/ Ntul//-cp (corpulent) rat
}

\begin{abstract}
Obesity develops in the obese phenotype of the congenic LA/Ntul//-cp (corpulent) rat strain by 6 weeks of age. ${ }^{1}$ To gain insight into the contributors to the expression of obesity in the obese phenotype of this strain, groups [n=12-20 rats/phenotype] of congenic male lean and obese LA/Ntul//-cp (corpulent) rats were fed an ad libitum standardized Purina chow diet (CHOW) from 6 to 12 weeks or age, and subgroups ( $\mathrm{n}=6$ rats / subgroup) were overfed with a highly palatable cafeteria diet (CAFÉ) from 9 to 12 weeks of age (WOA). A subgroup of obese rats $(n=6)$ were subjected to bilateral adrenalectomy (ADX) at 6 WOA and followed the same dietary regimen and treatment schedule. BW of lean and obese animals were similar at 6 WOA and increased by $88 \%$ in lean phenotype and $281 \%$ in obese phenotype during the 6 weeks study, while in ADX obese rats, BW were similar at 6 and 9 WOA but $\mathrm{BW}$ increased to 2.5 -fold above starting weights and 1.8-fold above 9-week weights between 9 and 12 WOA. The CAFE supplement was without significant effect on final body weights in the lean phenotypes, but was associated with significantly greater body weights at ages 9 and 12 WOA in the obese phenotype $(p=<0.05)$ and in the obese-ADX at 12 WOA. CE (kcal/gram gain of BW per day) remained relatively constant in lean and obese-ADX rats throughout the study, but $\mathrm{CE}$ was more efficient in the obese phenotype at all ages studied and was more efficient with the CAFE supplement feeding regimen. Fasting I:G ratios at 12 weeks of age were 4.2-fold greater in obese than lean and were partially normalized in obese-ADX to 1.7 -fold increase at 12 WOA. Relative adiposity of obese rats was 3.8-fold greater in obese than lean phenotype, with the greatest increase in the SQ depot. Resting VO2 (RMR) was lower in obese than lean rats at each age studied and was increased by ADX. Thermogenic interscapular brown adipose tissue (IBAT) mass was greater in obese and obese-ADX than lean rats. The results of this study indicate that $\mathrm{CE}$ is associated with the predisposition for the expression and development of adiposity in the obese phenotype of this strain and is associated with an increased I:G ratio and IBAT mass that is consistent with insulin resistance and an impaired capacity for energy expenditure and became normalized on the Chow but not the CAFE diet following ADX. These observations implicate likely multiple metabolic factors that contribute to a greater efficiency of energy storage, utilization and or energy conservation in the obese than in the lean phenotype of this strain and which is partially corrected in the obese phenotype by ADX. The metabolic impact of added caloric intake was associated with an additive impact on the $\mathrm{CE}$ of weight gain and adiposity in the obese phenotype of this congenic rodent strain and was partially corrected via ADX.
\end{abstract}

Keywords: caloric efficiency, obesity, brown adipose tissue, congenic rodent model
Volume II Issue 5 - 202I

\author{
Orien L Tulp, ${ }^{1-3}$ Aftab R Awan, ${ }^{1-3}$ George P \\ Einstein ${ }^{1-3}$ \\ 'University of Science Arts and Technology, Montserrat, the \\ British West Indies \\ ${ }^{2}$ The University of Health and Humanities, Virgin Islands, UK \\ ${ }^{3}$ The Einstein Medical Institute, FL, USA
}

Correspondence: Orien L Tulp, University of Science Arts and Technology, [US Office:Aspen Business Park, 5400 Ward Road, Building III, Suite 150-220,Arvada, CO, 80002. USA, Tel 0I-303-352-0II5, Fax 01-030-37I-0II7, Email o.tulp@usat.edu

Received: October 20, 2021 | Published: November 03, 202

\section{Introduction}

Epidemiologic reports indicate that obesity and overweight conditions are rapidly approaching epidemic proportions in Western society and are associated with increased risks for development of cardiovascular and other serious metabolomic-related comorbidities without a defined singular mechanism other than a dysregulation in energy balance. ${ }^{1,2}$ Moreover, the frequent consumption of energy dense palatable foods and their attendant impact on progression to overweight conditions are also approaching all-time highs proportions in younger ages than in the past in much of Western society. ${ }^{3-6}$ The development of insulin resistance is often considered to be a significant contributory factor in the progressive development of obesity, where it has also been linked to impaired thermogenesis and energy expenditure in brown adipose tissue and other tissues and a greater efficiency of energy deposition in adipose tissues. ${ }^{6,7}$ Cortisol concentrations and/or cortisol secretion rates have also been reported to be elevated in obesity but the metabolic link between elevations in cortisol and insulin are unclear. ${ }^{4}$ Recent studies in several rodent strains indicate that introduction of a palatable diabetogenic diet may predispose to insulin resistance and energetically more favorable lipid deposition and gender specific regionalization of adiposity in female vs. male animals and in genetic models of obesity fed the high energy diets. ${ }^{1,7}$

The effects of genetic predisposition as an independent variable in the development of obesity however, is more difficult to assess in human populations due at least in part to the wide genetic variability present in the general population, and different dietary and exercise patterns undertaken in different segments of the population. The LA/Ntul//-cp (corpulent) rat is a congenic animal model, developed from the LA/N strain at the NIH by Hansen ${ }^{8}$ and Greenhouse ${ }^{9}$ and further characterized by Michaelis ${ }^{10}$ and Tulp. ${ }^{1,7}$ Obesity occurs in this strain as the result of an autosomal recessive trait, and thus appears 
in $\sim 25 \%$ of the multigenerational offspring of heterozygous breeding pairs for the (-cp) trait. ${ }^{1,8,9}$ Obese offspring have rarely been observed to reproduce due to endocrinopathies that as yet remain unclear. ${ }^{1}$ The obese phenotype demonstrate aberrations in circulating plasma insulin, amylin, and lipid concentrations and in energy expenditure, while remaining normotensive, and are modestly glucose intolerant but remain non-diabetic throughout adulthood. ${ }^{11-13}$ The current animals were derived following the $12^{\text {th }}$ backcross at the NIH by Hansen thereby establishing the congenic criterion for the colony and virtually eliminating experimental variability within the LA/Ntul//-cp strain with the exception of the expression of the $(-c p)$ characteristic in the obese phenotype. ${ }^{8,9}$ The feeding of highly palatable cafeteria diets and high fat diets have also been linked to greater adiposity and more efficient weight gain in the obese phenotype in female corpulent rats as well as in other rodent strains.?

Because the obesity phenotype is expressed as an autosomal recessive trait it occurs with great multigenerational consistency in the offspring of heterozygous breeders, it is thus an excellent animal model to investigate the independent variables of age and phenotype on metabolic factors likely to be contributory or causative to the predisposition of obesity and overweight conditions independent of NIDDM. ${ }^{5,6}$ The LA/N background strain was noted for its longevity by Hansen ${ }^{8}$ and in studies by Tulp indicate that this strain ranks among the most long-lived of the obese rodent models yet reported, with some animals surviving beyond 4 years and obese animals over 3 years under standard laboratory conditions. ${ }^{1,7}$ Metabolically, lean animals have normal thyroidal and thermogenic responses to resting oxygen consumption (RMR), and to dietary intake and cold environment, ${ }^{10,11}$ while the dietary and environmental responses in obese animals with the (-cp) trait have consistently been shown to be impaired under such conditions. ${ }^{1,7,12}$ The presence of insulin resistance in the expression of impaired thermogenesis in concert with an enhanced propensity for adiposity is well established, while the role of adrenal corticoid hormones are believed to exert only a permissive role in energy expenditure and storage, while conditions of hyperinsulinemia and insulin resistance impair the thermogenic responses. ${ }^{6,7}$ Several studies have indicated beneficial effects on energy metabolism following ADX but the effects of ADX on caloric efficiency or energy expenditure in this strain have not previously been reported. Numerous studies indicate that carbohydrate rich and energy dense dietary supplements may enhance net energy intake, often associated with greater energy deposition and thermogenic energy expenditure ${ }^{13}$ in normally lean rats, but result in greater adiposity in the obese phenotype of this and other genetically obese rat strains. ${ }^{14-18}$ Schlafani et al investigated a broad range of dietary carbohydrate sources and reported that introduction of a $16 \%$ sucrose solution $(\mathrm{w} / \mathrm{v})$ as a drinking water supplement could stimulate adaptive thermogenesis in lean rats with only a minimal impact on overall caloric intake, ${ }^{19-22}$ and Stock and Rothwell ${ }^{14}$ and Tulp et al., ${ }^{1,23,24}$ found that $\mathrm{CHO}$ enrichment resulted in increases in energy expenditure mechanisms in lean but not in obese rats. Tulp and Carlin ${ }^{25}$ demonstrated that the complex CHO Polycose, a form of partially hydrolized cornstarch, resulted in greater energy intakes and adiposity than the sucrose solutions referred to above in this strain suggesting that the perceptions or preferences for sweetness in rats may differ from those as perceived by humans. Thus, the LA/N//-cp rat approach presents a valuable tool to enable one to assess the impact of modest caloric supplementation on diet induced thermogenesis and caloric efficiency while exerting minimal effects on body composition in normally lean animals. ${ }^{1,7,8,10}$

The combined effects of multiple peripheral tissues including liver, muscle and brown adipose tissue contribute to mechanisms and processes of energy metabolism and expenditure in mammalian species. Brown adipose tissue [BAT] is a major thermogenic tissue of man and animals ${ }^{21}$ and can be stimulated by $\mathrm{CHO}$ feeding, cafeteria overfeeding, cold exposure and noradrenergic stimulation via $\beta 3$ adrenergic agents. ${ }^{18-20}$ In mammalian animals, including humans, BAT normally becomes activated soon after birth and is responsible for heat generation via expression of uncoupling protein-1 (UCP-1) and resultant uncoupling of oxidative phosphorylation, which generates heat from ATP to ADP high energy P bonds and thus assists in maintaining body temperature during the early life of the newborn of the species, as well as becoming active during periods of overnutrition and cold exposure. ${ }^{21}$ BAT depots remain histologically present well into adulthood in many mammalian species studied. Histologically, brown adipocytes differ markedly from white adipocytes on the basis of their smaller cell size, numerous specialized mitochondria, a centrally located nucleus, and multiple small lipid locules, in addition to sympathetic neuroregulation via $\beta 3$-adrenergic receptors. In contrast, white adipocytes contain a larger single lipid droplet focused on mechanisms of energy deposition and storage, a small peripherally located nucleus within a cytoplasmic ring surrounding the adipocyte lipid droplet, fewer mitochondria, and lack direct neural or $\beta 3$-adrenergic stimulation. ${ }^{18-20,22}$

The lipid locule size of adipocytes is believed to play an important role in BAT mediated energy expenditure as the smaller lipid locule size in BAT vs White adipose tissue (WAT) adipocytes results in a greater net surface area to lipid mass, in the smaller lipid droplets thereby enabling a more efficient process of fatty acid mobilization, oxidation and resultant heat generation than is possible in WAT adipocytes. ${ }^{26-36}$ BAT has been proposed as contributing factor to an energy balancing mechanism for energy balance especially during caloric overnutrition ${ }^{21}$ while in addition to thyroidal mechanisms that have been demonstrated to respond to both over- and undernutrition by switching from outer ring $\mathrm{T} 4$ deiodination from the active form (T3) during abundant caloric availability and cold exposure to the inner ring inactive form, reverse T3 (rT3) during periods of caloric restriction, food depravation or starvation. ${ }^{16}$ The T3, as the metabolically active form of the thyroid hormone binds to nuclear receptors and presumably contributes to expression of a number of metabolic processes including rates of protein synthesis and degradation (protein turnover), carbohydrate oxidation, and other processes including the energy wasting hepatic mitochondrial $\alpha$-glycerophosphate dehydrogenase shuttle, which has been shown to correlate with circulating T3 concentrations ${ }^{37}$ and to diminishing activity levels with advancing age in rats. ${ }^{1,38,39}$ The metabolism of the $\alpha$-glycerophosphate shuttle was also reported to be decreased in subcutaneous adipose tissue samples obtained from adult obese subjects. ${ }^{39}$

When brown adipocytes are metabolically active the lipid locules become decreased in size, while in the presence of sympathetic blockade, the lipid locules were found to be of larger cross sectional diameter, indicative of decreased BAT thermogenic activity. ${ }^{25}$ In addition, BAT develops chronologically via hyperplasia early in life, while WAT adipocytes increase by hyperplasia and hypertrophy throughout much if not the entire life span in man and animals particularly in subcutaneous depots where adipocyte hyperplasia and hypertrophy are likely to experience fewer internal or abdominal constraints on expansion. ${ }^{15,32}$ Once formed, the BAT mass formed in early life in rodents has been shown to remain present well into adulthood, and in an enhanced capacity for NE-stimulated energy expenditure in lean Sprague-Dawley rats. In studies in normally lean rats, early overfeeding was observed to increase both brown adipocyte 
number and total interscapular depot mass, and which increased mass may remained present into adulthood of the rat, with the result of a greater potential capacity for noradrenaline and cold stimulated thermogenesis later in life. ${ }^{1,32}$ IBAT activity is well established to be a physiologic component of diet induced thermogenesis [DIT] in man and animals. ${ }^{7}$ Whether nutritionally an environmentally increases in IBAT number and activity may occur in adulthood in animals and man is unknown, but recent studies suggest that some transdifferentiation between white adipocytes and brown adipocytes may occur, forming beige adipose tissue, a third possible type of adipose tissue also with a thermogenic capacity. ${ }^{33}$ Other biochemical and physiologic mechanisms also likely contribute to buffer variations in energy metabolism, including neuroendocrine, thyroidal, pancreatic, hepatic, and other metabolic processes. ${ }^{6}$ Historically however, most rodent studies have been conducted in younger male animals, and may not therefore correlate fully with the physiologic and neuroendocrine changes that occur in aging and or which may occur in males vs. females as a normal consequence of early development at least in part due to gender and age related endocrinologic differences. The purpose of this study was to assess the effect of ADX and indirect measures of insulin resistance in the male obese phenotype on CE and adiposity and to determine if the impaired responses to diet in the obese phenotype were associated with corresponding changes in $\mathrm{CE}$ which might be correlated with the greater adiposity often associated with the $(-c p)$ trait.

\section{Materials and methods}

Animal Selection. Groups [n=12-20 rats/group] of congenic, specific pathogen free (SPF) male lean and obese littermate LA/Ntul//$c p$ rats were selected from the Drexel University breeding colony at 6 weeks of age and maintained in shoebox plastic cages with pine shavings with a reverse light cycle [dark cycle $0800-2000 \mathrm{~h}$ daily] in a temperature-controlled environment $\left[22+/-1^{\circ} \mathrm{C}\right]$ and $40-60 \%$ relative humidity from 6 weeks of age. Animals were fed standard ground Purina chow \# 5012 with a manufacturer's reported energy density of $3.34 \mathrm{kcals} / \mathrm{gram}$ and house water ad libitum throughout the study or the same ration plus a palatable cafeteria diet supplement from 9 to 12 weeks of age as described previously.(ref) All housing and laboratory procedures were in accordance with current NIH, AVMA and Institutional Animal care and Use Committee guidelines. Except for the obese trait, biologic variability between offspring of different litters is virtually absent due to the congenic status of this strain and is limited to the potential heterozygosity within the lean littermates of the strain, some of which may carry a homozygous lean trait (-$/--)$ and some a heterozygous $(-/-c p)$ lean trait. In contrast, all obese littermates are homozygous $(-c p /-c p)$ for the obese characteristic. All breeding pairs consist of heterozygous carriers for the obese trait.

Food and Energy Intake. Measures of 24-hour food intake were obtained in individual animals placed in metabolic cages over duplicate 3-day periods, and the data collected for record from the second 3-day period to allow for animals to adequately acclimatize to the metabolic cages. Weights of food consumed including cafeteria food items were determined before and after on an electronic balance to the nearest 0.1 gram and corrected for spillage. Food intake is expressed as $\mathrm{kcals} / \mathrm{rat} / \mathrm{day}$ including the actual amounts of cafeteria foodstuffs and chow consumed. Computation of caloric efficiency was determined by measuring the total kcals of food consumed/gram of body weight gain per day as reported by Vedula et al. ${ }^{36}$ Measures of animal body weight were obtained with an Ohaus animal balance to the nearest gram at weekly intervals. Statistical analysis was computed with a $2 \times 3$ ANOVA and students ' $t$ ' test with the Student Newman Keuls application to determine significance level in subgroups where more than 2 groups are compared..$^{37}$ Descriptive statistics identified outliers within each group and values more than 2 standard deviations from the mean were eliminated from the statistical analysis, and statistical significance was set at alpha of $\mathrm{p}<0.05$.

The Café diet items selected included measured portions of common dietary foodstuffs each with the metabolizable energy (as $\mathrm{kcal} / \mathrm{gram}$ ) as reported by the manufacturer as follows:

The Chow and Cafeteria Diet constituents. Purina Chow (3.34), Marshmellows (3.3), Candy Corn (3.6), Peanut Butter crackers (4.3), Peanut Butter Cookies (4.0), Kielbasa (3.1), Life cereal (4.2), Cracker Jacks (4.2), Cheese Doodles (5.3), Oreo Cookies (4.9), Chocolate Cookies (5.0), White Bread (2.9), Pretzels (4.0), Shortbread (5.3), Hot Dogs, uncooked (3.2), Pudding (1.2), Animal Crackers (4.3), Granola (3.2), Peanut Butter Cups (5.4), Chocolate Kisses (5.3), and Froot Loops (3.9). Each animal was provided with 4 cafeteria food items each day from weeks 9 to 12 in an attempt to induce hyperphagia via greater palatability, and thereby potentially exaggerate the nutritionally induced thermogenic responses. While peanut butter items proved to be among the more favoured snack items by most rats, all of the above were enthusiastically consumed in generous quantities in addition to normal quantities of the Purina chow diet. A hallmark of the items included in the cafeteria food items are that most items offered have energy densities that are greater than the Purina chow ration, and most have greater carbohydrate and lipid content and greater sweetness than the Purina chow. All animals were provided the same café selections as all other animals of each treatment group each day.

Resting Metabolic Rates. Measures of Resting metabolic rate (RMR) were determined in lean, pre-obese and pre-obeseadrenalectomized animals at 6 weeks of age with Collins small animal spirometer apparatus to aid in the selection of obese vs lean rats as described previously. ${ }^{1,7,19}$ The apparatus was fitted with a 1 cubic foot volume sealable Plexiglas chamber large enough for the animals to move around, rear or rest comfortably. The chamber was charged with 1 liter of room air, corrected for atmospheric pressure and altitude, and air circulation was maintained throughout 30 minutes of continuous observation with a veristaltic pump, and the temperature of the chamber was maintained at $30^{\circ} \mathrm{C}$ by placement in a temperature controlled circulating water bath. Moisture and $\mathrm{CO}_{2}$ were removed from the system with chemical absorbent filters containing Drierite and Soda Lime, respectively, and strategically incorporated into the circulating air lines and changed between animals insure efficiency of moisture and $\mathrm{CO}_{2}$ removal. Animals were placed in the sealed $\mathrm{VO}_{2}$ chamber, the system sealed, and measurements of $\mathrm{VO}_{2}$ taken after 15 minutes to allow the system to fully equilibrate, and are expressed as $\mathrm{ml}$. oxygen consumed per minute per $\mathrm{kg}$ of body ${ }^{-0.75}$ to allow for differences in body surface area as described by Klieber and Huang et al. ${ }^{38,39}$ and observation of the animals during the process indicated that after a brief one to two minute period of exploration, animals were observed to rest comfortably throughout the remainder of the $\mathrm{VO}_{2}$ process. Additional measures of RMR were obtained after 2, 4 and 6 weeks of the study.

Adrenalectomy in pre-obese animals. Pre-obese animals were subjected to bilateral adrenalectomy at 6 weeks of age upon confirmed establishment of phenotype via measures of $\mathrm{VO}_{2}$ and physical characteristics. The surgical procedure was performed under pentobarbital anesthesia $(5.5 \mathrm{mg} / \mathrm{kg}$ body weight, i.p.) as outlined by Marchinton et al., ${ }^{25}$ with the animals in the prone position, with a draped heating pad underneath to minimize heat loss during and after the anesthesia. The adrenals were located via single a $1 \mathrm{~cm}$. 
mid-posterior incision and removed in their entirety. During recovery and immediately thereafter animals were provided ad libitum access to a $0.9 \% \mathrm{NaCL}$ solution in addition to normal house water. The surgical procedures were conducted upon inclusion to the study and assignment to a treatment group and prior to the development of overt obesity, and while containing the Chow diet described above. All animals survived the surgical procedure and subsequent observations and treatment regimen without incident.

Determination of I:G ratios. At the end of the study, fasting bloods were obtained and measures of fasting blood glucose and insulin determined. Blood glucose was determined via a glucose oxidase procedure, and serum insulin via radioimmunoassay as described previously. ${ }^{12,13}$

Adipose Tissue Dissection. At the end of the study, rats were sacrificed by rapid, humane decapitation and selected anatomically defined adipose tissue depots carefully dissected in their entirety and weighed on an electronic balance and recorded to the nearest 0.1 gram. The specific depots dissected were the retroperitoneal, epididymal, dorsal, and interscapular brown adipose tissue depots. Abdominal depots were dissected with the animal in the prone position, and the dorsal subcutaneous and IBAT depots dissected with the animal in the supine position.

\section{Results}

The body weights of animals at 6,9 , and 12 weeks of age are depicted in Table 1 below and indicate that the final live body weights of lean animals increased an average of 23 grams per week, and the rate of weight gain was minimally impacted by addition of the cafeteria diet in weeks 3 to 6 when aged 9 to 12 weeks of age of the study. In contrast, in obese rats the rates of weight gain were approximately twice that observed in the lean phenotype when fed the Chow or the CAFÉ diet regimen. Adrenalectomy normalized the rate of weight gain in the obese phenotype to that observed in the lean animals during the 3-week chow only segment of the study, but the rate of weight gain in obese-ADX rats during the cafeteria diet phase was similar to that observed in lean rats during the Chow only segment of the study but increased at a rate similar to that observed in unoperated obese rats serving as an obese control group during the final 3-week CAFÉ + chow segment of the study. Preliminary Resting metabolic rates $\left(\mathrm{RMR}, \mathrm{VO}_{2}\right)$ are depicted in Figure 1 with data obtained at 6 weeks of age and indicate that the RMR of lean rats was greater than that observed in obese rats by 6 weeks of age and was a contributing predictor of phenotype prior to overt expression of the developing obese state.

Table I Body weights of lean, obese and obese-adrenalectomized rats

\begin{tabular}{llllll}
\hline Group,Age & N & 6 Weeks & 9 Weeks & 12 Weeks & Net gain 6- I2 W \\
\hline Lean & 6 & $155.7 \pm 4.6 \mathrm{a}$ & $225.5 \pm 8.4 \mathrm{a}$ & $292.5 \pm 8.8 \mathrm{a}$ & $137 \pm 7.3 \mathrm{a}$ \\
Obese & 6 & $158.2 \pm 17.3 \mathrm{a}$ & $303.8 \pm 20.0 \mathrm{~b}$ & $446.3 \pm 24.9 \mathrm{~b}$ & $276.1 \pm 20.4 \mathrm{~b}$ \\
Obese-Adx & 6 & $166.2 \pm 13.2 \mathrm{a}$ & $236.2 \pm 8.8 \mathrm{a}$ & $424 \pm 5.9 \mathrm{~b}$ & $238.1 \pm 9.3 \mathrm{~b}, \mathrm{c}$
\end{tabular}

Data are mean \pm I SEM. Letters indicate significant different groups at $p=<0.05$ via Student-Neuman-Keuls. Rats received a Purina Chow diet (stock nr 50 I2) from 6 to 9 weeks of study, and the Chow diet plus a palatable cafeteria diet from 9 to 12 weeks of age

\section{RESTING METABOLIC RATES OF LEAN AND OBESE PHENOTYPE}

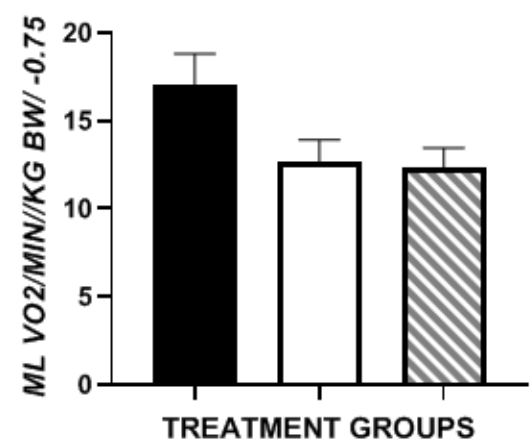

\author{
LEAN \\ 口 OBESE \\ D PRE-OBESE+ADX
}

Figure I Data are mean RMR +/- I SEM, N= 6 rats/phenotype and treatment group.All measures performed at thermal neutrality $\left(30^{\circ} \mathrm{C}\right)$ in the fasting state.

Energy intake of animals is depicted in Figure 2 in weeks 2 (chow only) and weeks 4, 5 and 6 (Chow+CAFE) and caloric efficiency of weight gain in Figure 3. The data reflect the combined total of kcals consumed as chow only or as Chow plus CAFÉ during the final 3 weeks of the study. Energy intake in the lean phenotype remained similar throughout the study, with only a modest increase when offered the CAFÉ supplement in weeks 6 to 9 of the study. Energy intake of obese and obese-ADX animals was consistently greater in obese than in lean animals throughout the study, weather expressed in absolute terms as kcals/rat/day (Figure 2) or relative to body weight as caloric efficiency (Figure 3). In lean animals, the efficiency of weight gain decreased with age as the animals grew from a mean of
$0.083 \mathrm{~g}$. gain $/ \mathrm{kcal}$ consumed on the chow diet during week 2 (age 7-8 weeks) to $0.0193 \mathrm{~g} . / \mathrm{kcal}$ consumed in week 6 (age 12 weeks), when most of their lean tissue and linear growth was likely attained. The metabolic efficiency of weight gain among obese animals although similar to that observed in lean animals at each age studied tended to be greater and more efficient, despite their rapid lean growth phase. In contrast, the efficiency of weight gain in the obese phenotype when adrenalectomized was intermediate between that observed in lean and obese animals from 4 weeks of age, and unlike the lean and unoperated obese, $\mathrm{CE}$ remained constant at each age measured throughout the study. 
EFFECT OF ADX AND DIET ON DAILY CALORIC INTAKE Diet:Week 2: CHOW/Week 4 - 6: CHOW+CAFE

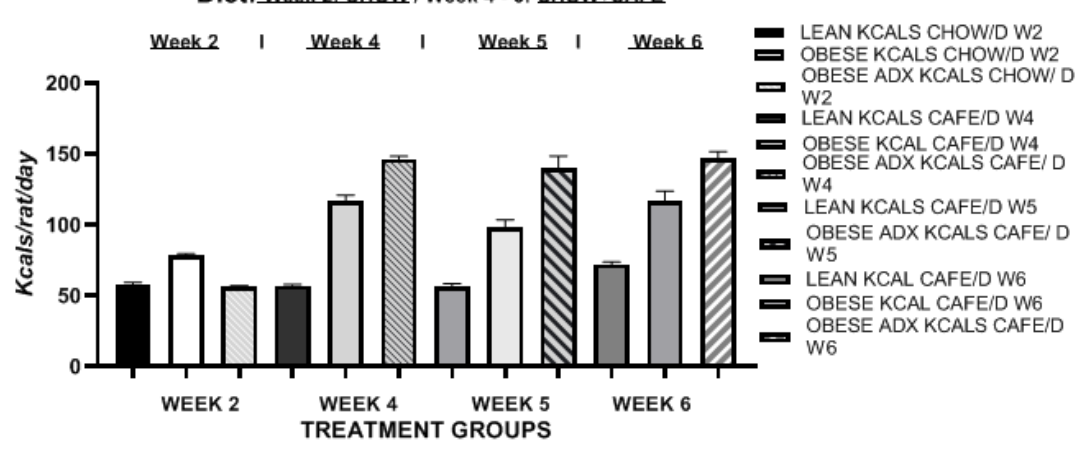

Figure 2 Effect of phenotype and adrenalectomy on caloric intake in LA/Ntul//-cp rats. Data are mean \pm I SEM, $n=6$ rats/treatment group. Rats consumed the chow diet in week 2, and the chow + the cafeteria diet in weeks 4,5 and 6, corresponding to rat ages of 8, I0, II, and I2 weeks, respectively depicted from left to right.

\section{EFFECT OF PHENOTYPE AND ADX ON FEED EFFICIENCY}

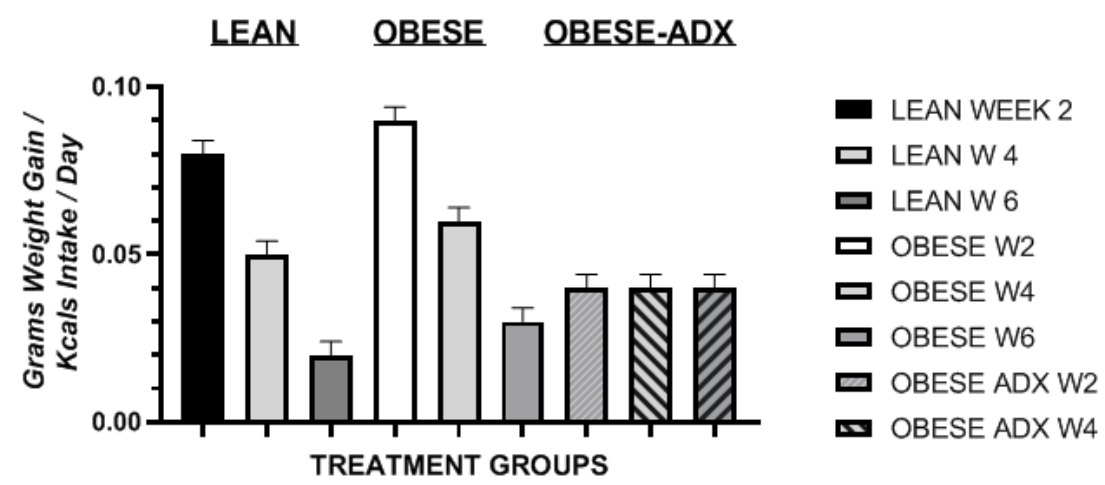

Figure 3 Effect of phenotype and adrenalectomy on caloric efficiency in LA/Ntul//-cp rats. Data are mean \pm I SEM, n=6 rats/treatment group. Rats consumed the chow diet in week 2 , and the chow + the cafeteria diet in weeks 4,5 and 6 , corresponding to rat ages of 8 , I0, II, and I 2 weeks, respectively depicted from left to right. The feed efficiency, i.e. caloric efficiency, was computed as the kcals of energy consumes per gram of weight gain per day as outlined previously. ${ }^{34}$

White Adipose tissue (WAT) mass in the retroperitoneal and epididymal abdominal depots and in the dorsal subcutaneous depots were dissected in their entirety at the end of the study (age 12 weeks) and depicted in Figure 4 and indicate that the combined RP+EPI mass of lean animals was significantly greater than that dissected in the SQ WAT depot. In contrast, both SQ and Abdominal WAT depots were signifancantly greater in the obese phenotype than the lean phenotype fed the same dietary regimen, with the greatest increase observed in the SQ WAT depot. In contrast, in obese-ADX rats, the combined Retroperitoneal + Epididymal abdominal WAT depot mass was similar to that observed in lean animals, and the marked increase in SQ WAT of obese-ADX rats compared to lean rats consuming the same diet was significantly greater but only half that of similarly fed obese rats, likely reflecting an amelioration of adreocorticoid influence on the efficiency of lipid accretion and weight gain when compared to unoperated obese animals consuming equivalent quantities of the same diet under the same environmental conditions. This observation suggests that the biochemical processes of lipid accretion may favor the accumulation of subcutaneous fat over abdominal fat in rats of this age and gender and corresponding to an age when the animals would have attained near full maturity.

\section{EFFECT OF ADX ON ABDOMINAL \& SUBCUTANEOUS ADIPOSE TISSUE MASS}

\section{LEAN OBESE OBESE-ADX}

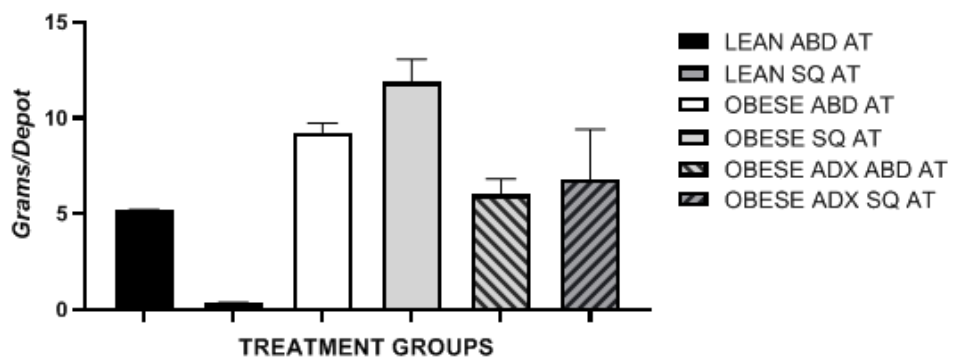

Figure 4 Adipose Tissue Depots in lean, obese and obese+adrenelctomized rats. Data are mean $\pm I S E M, N=6$ rats per treatment group. Abdominal depots depicted were the sum of the retroperitoneal and epididymal fat depots, and the subcutaneous depicted as the sum of the dorsal fat depot. 
Net WAT accumulation in both abdominal and subcutaneous depots consuming the same diets since birth at 12 weeks of age as depicted in Figure 5 suggest a strong association with the serum Insulin to Glucose ratios depicted in Figure 6 and illustrate a strong connection between WAT accumulation and insulin to glucose ratios, regarded as a measure of insulin sensitivity, and associated lipid accretion in the WAT depots. Net WAT mass and I:G ratio were lowest in the lean animals and increased up to 4-fold in the obese rats. In contrast, the I:G ratio of obese-ADX rats approached those observed in lean animals (1.4-fold greater), and the net WAT mass of obese ADX animals was intermediate between the lean and unoperated obese rats undergoing the same dietary regimen and consuming similar energy intake throughout the study.

\section{EFFECT OF ADX ON INSULIN : GLUCOSE RATIO}

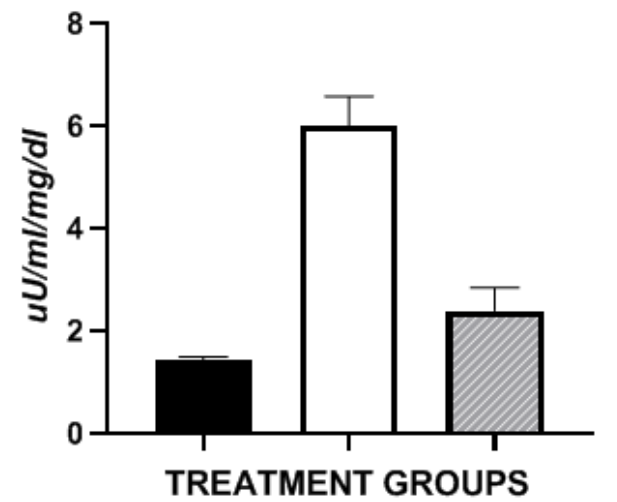

- LEAN I:G

口 OBESE I:G

OBESE ADXI:G

Figure 5 Fasting insulin to glucose ratios of lean obese ad obese-ax rats. Data are mean \pm I SEM, N= 6 rats/group. Insulin concentrations Insulin concentrations were recorded as $\mu \mathrm{U} / \mathrm{ml}$ and glucose as $\mathrm{mg} / \mathrm{dl}$.

RESTING VO2 IN LEAN, OBESE AND OBESE-ADX RATS

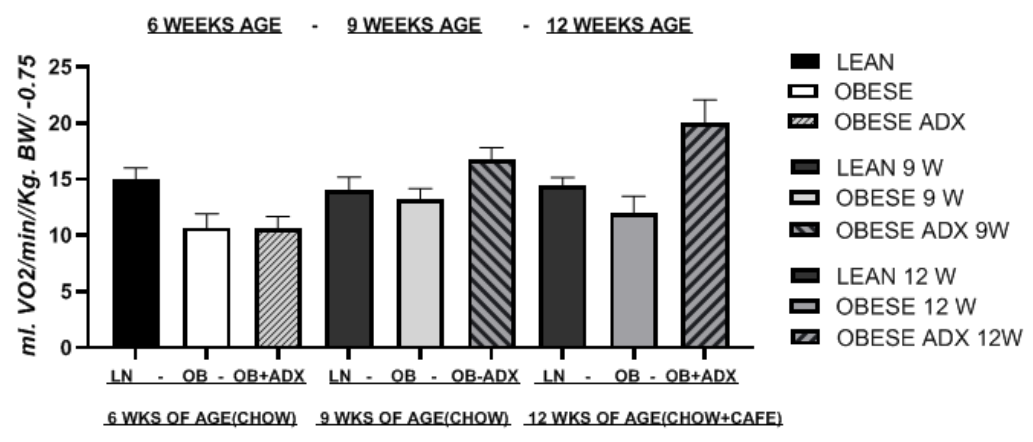

TREATMENT, AGE AND DIET GROUPS

Figure 6 Effects of phenotype diet and adrenalectomy on resting metabolic rates in fasted lean and obese LA/Ntul//-cp rats. Data are mean \pm I SEM, $\mathrm{n}=6$ rats/ treatment group.All measures obtained at thermal neutrality $\left(30^{\circ} \mathrm{C}\right)$.

Measures of RMR throughout the study are depicted in Figure 6 and indicate that the RMR of lean animals remained greater than in obese animals at each time measured. The RMR of animals of each treatment group at 6 weeks of age while consuming the chow diet are depicted in the left 3 bar panel, from 9 weeks old animals of each treatment group receiving the chow diet in the central 3-bar section, and from 12-week-old animals receiving the cafeteria plus chow regimen in the right 3 bar section of Figure 6 . These data show that $\mathrm{VO} 2$ of lean rats consuming either dietary regimen were greater than in obese rats consuming the same dietary regimen at 6,9 and 12 weeks of age. Adrenalectomy in the obese phenotype resulted in a restoration of VO2 to that observed in their lean littermates at 9 weeks of age and the VO2 increased further after consuming the Cafeteria diet supplement in the 12 week data. Thus, adrenalectomy resulted in increases in RMR to levels similar to the VO2 of observed in lean littermate animals of the same age but consuming greater quantities of the stock chow at 9 weeks of age, and increased to even greater $\mathrm{VO}_{2}$ readings at 12 weeks of age after consuming the chow plus the cafeteria diet regimen, as depicted in the right 3 bars of Figure 6.
Thus, the results are consistent with the proposal that adrenal cortical secretions, likely the glucorticoids cortisol and / or corticosterone common to adrenal secretions in the rat and other mammalian species, contribute to the development of insulin resistance in the obese phenotype, and which phenomena results in or contributes to an impaired expression of thermogenesis, caloric efficiency and fat accretion that is a characteristic hallmark of the obese state. Moreover, this dysregulation of energy metabolism emerges early in the development and lifespan of the LA/Ntul//-cp rat.

\section{Discussion}

Some of the contributory and counterregulatory roles of insulin and adrenal corticoid hormones on parameters of energy metabolism, energy expenditure and lipid accretion are well established. ${ }^{4}$ Insulin permits both lipid biosynthesis in adipose depots and decreases in protein degradation in lean tissues, thereby contributing to an efficiency of energy utilization via an attenuated rate of protein turnover, and shuttling substrates into dietary sources and both de 
novo lipid biosynthesis and dietary lipid deposition, both energetically more efficient processes than oxidation of most substrates. Protein synthesis, at consumption of up to 4 ATPs per peptide bond formed, represents one of the most energetically expensive processes of normal growth, development and metabolism. ${ }^{1}$ In addition, when protein sources are metabolized as an energy source, they yield considerably less metabolizable energy than lipid sources $(\sim 4 \mathrm{kcal} /$ gram vs $\sim 8 \mathrm{kcal} / \mathrm{gram})$. Thus, the gradual increases in plasma insulin concentrations during early growth have the potential to shift energy metabolism into more energetically conservative states. Indeed, when obese Zucker rats and LA/Ntul//-cp rats were fed a protein restricted diet, containing only $8 \%$ of the diet as protein, protein restricted animals of both strains grew normally, attaining normal body size and lean tissue grown that was indistinguishable from that attained by normally fed obese animals of either strain consuming diets containing $24 \%$ protein by weight. ${ }^{16,38}$ The present study was conducted during an early and active stage of growth and development, when lean tissue and linear growth were occurring at heightened energy cost to the growing animal. Yet in the presence of elevated plasma insulin concentrations, the insulin-mediated effects on protein degradation and lipid biosynthesis became evident by 9 weeks of age while consuming the nutritionally adequate chow diet or at 12 weeks of age, while consuming the lipid and carbohydrate energy-rich rich palatable cafeteria dietary regimen in addition to the chow diet. Of interest, both lean and obese animals fed the chow+café diet continued to consume significant quantities of the chow ration, thereby enabling lean tissue growth to occur at a normal rate.

Taste preferences for sweetness likely differ between rats and humans however, particularly with respect to simple vs. complex carbohydrate sources, with rats demonstrating a preference for complex starches such as are present in polycose, a partially hydrolyzed form of cornstarch vs the simple carbohydrate sucrose, where animals given the choice of either consumed considerably larger quantities of the polycose than the sucrose solution..$^{8,25}$ Nonetheless, the broad variety included in the cafeteria diet outlined above offered numerous tastes that proved effective in inducing hyperphagia in both lean and obese animals in this study, including adrenalectomized obese animals. Obese animals of both unoperated and adrenalectomized groups readily adapted to the chow plus cafeteria feeding regimen as depicted in Figure 2, with the adrenalectomized animals consuming the greater quantities of the dietary offerings. Whether this was due to the sweetness or overall palatability of the diets in those groups, or to the greater salt content was unclear but is likely to have played a contributory role since those animals would also have been deficient in aldosterone in addition to the glucocorticoid hormones. In unoperated obese animals the café diet induced significant hyperphagia.

The efficiency of weight gain, reported as caloric efficiency, tended to be greater in the obese than the lean phenotype at each age determined, with more weight being gained per kcal consumed in the obese phenotype than the lean phenotype, suggestive of a more efficient net metabolism in the obese animals (Figure 3) similar to that reported in aging female rats previously. ${ }^{7}$ In contrast, the caloric efficiency of weight gain in adrenalectomized obese animals was similar to that observed in lean animals when fed the chow only diet but was enhanced when fed the café+cafeteria regimen with more kcals consumed per gram of weight gain and likely reflecting a greater thermic response in the presence of hyperphagia. Resting oxygen consumption (RMR) obtained at multiple intervals during the study confirmed the greater resting energy expenditure in lean vs obese animals at each age studied, and that adrenalectomy in the obese animals was without effect when fed the chow only diet, but restored RMR to values equal to or greater than were observed in the lean phenotype during weeks 4 to 6 of the study when significant hyperphagia was present, and when adrenal corticoid hormonal effects were likely absent due to the adrenalectomies performed 4 to 6 weeks previous. No measures of fed intake or caloric efficiency were obtained during week one of the study, deemed a transitional week while adrenalectomized animals may have experienced remnants of surgical recovery but by week two following surgery residual effects of their prior surgery on parameters of food intake or caloric efficiency were no longer apparent.

Patients with abdominal obesity have elevated plasma cortisol and insulin levels. Furthermore, stress and glucocorticoids act to control both food intake and energy expenditure. In particular, glucocorticoids are known to be associated with increased trends toward consumption of foods enriched in fat and sugar, likely to increase insulin and triglyceride levels. ${ }^{41-43}$ The insulin to glucose ratios of obese animals of the present study are also consistent with early onset of hyperinsulinemia and insulin resistance in peripheral tissues, and the measurement of the plasma insulin concentrations at 12 weeks of age confirmed that fasting insulin concentrations were 4-fold greater in obese than in lean animals, despite consuming the same diet and as their lean littermates of the same age and genealogy were consuming, and the animals of both phenotypes were genetically identical except for the presence and expression of the $-c p$ trait. Marchington et al reported that adrenalectomy improved energy expenditure and partially reversed the development of obesity in obese Zucker rats ${ }^{26}$ similar to that which was observed in the present study. The demonstration of near full recovery of insulin to glucose ratios in adrenalectomized obese rats further supports the hypothesis that the counter regulatory effects of the glucorticoid hormones contribute at least in part to the hyperinsulinemia, insulin resistance, and impaired thermogenesis of obesity. In humans, cortisol secretion rates and peripheral kinetics are known to become altered in the obese state, with obese individuals secreting significantly more cortisol than occurs in leanness, also with less variation in the diurnal plasma concentration patterns such that the mean cortisol concentrations remain higher than normal and partially elevated during parts of the day. ${ }^{40} \mathrm{Kahle}^{39}$ and Michaelis ${ }^{10}$ reported elevated cortisol levels in obese LA/N-cp rats, consistent with the observations of the present study. Thus, the counterregulatory impact of adrenal cortical hormones and its attendant impact on insulin actions at the receptor level enhance metabolic processes such as glucose and amino acid uptake and oxidation, protein degradation and protein synthesis in peripheral tissues, resulting in a greater economy of amino acid and glucose substrate utilization in the obese phenotype of this and likely other obese rodent strains. The luminal digestion and absorption, and the intermediary metabolism of proteins and carbohydrate sources occur similarly in rodents and humans and thus the implications of this study may also have application to the efficiency of energy metabolism in mammalian species other than rodents. The epidemic of obesity continues to be a global issue in both developing and developed counties where economic affluence and dietary preferences may enable access to and abundancy of highly palatable food choices and contribute to overweight and obese conditions and the attendant pathophysiologic sequelae which may follow, particularly in those individuals who may be genetically predisposed to easy weight gain through no fault of their own. ${ }^{43}$

\section{Acknowledgments}

The author wishes to acknowledge the contributions of Dr. Stephen Dubin, Mr Huang Peisong and Ms Mary Victor of Drexel University for veterinary assistance, animal husbandry and animal care assistance respectively, Caroline Sandler MS for the data collection and analysis, and the late Dr. Otho E Michaelis IV, of the Carbohydrate Nutrition 
Research Laboratory, Beltsville, MD, USDA for contributions during the planning and completion of this research.

Support: Supported by institutional resources of USAT, UHHVI and the Einstein Medical Institute.

\section{Conflicts of interest}

The authors declare that they have no competing interests.

\section{Funding}

None.

\section{References}

1. Tulp OL. Characteristics of thermogenesis, obesity, and longevity in the LA/N-cp rat. ILAR News. 1990;32(3):32-39.

2. Jacks DG, Kerna NA. A Comprehensive Analysis of Obesity Part 1. Overview of Obesity. J Obese Nutr Disord. 2018.

3. Björntorp Per, osmond, Roland MD, Obesity and Cortisol. Nutrition. 2000;16(10):924-936.

4. Centers for Disease Control and Prevention: National Health and Nutrition Survey, 2013-2014.

5. Kelly T, Yang W, Chen CS, et al. Global burden of obesity in 2005 and projections to 2030. Int J Obes (Lond). 2008;32(9):1431-1437.

6. Michael S Rahman, George P Einstein, Orien L Tulp. Autonomic, Immunological and Endocrine influences on adipose tissue as an organ Adv Obes Weight Manag Control. 2021:11(2):48-58.

7. Tulp, OL Effects of aging, phenotype and carbohydrate feeding on caloric efficiency and adiposity in the LA/Ntul//-cp (corpulent) rat. Adv Obes and Weight Manag and control. 2021;11(1).

8. Hansen CT. The development of the SHR/N- and LA/N-cp (Corpulent) Congenic Rat Strains. In: New Models of Genetically Obese Rats for Studies in Diabetes, Heart Disease, and Complications of Obesity. NIH publication, Division of Research Services, Veterinary Resources Branch, National Institutes of Health, Bethesda, MD. 1988. p. 7-10.

9. Greenhouse DD, Hansen CT, Michaelis OE IV. Development of Fatty and Corpulent Rat Strains. In: New Models of Genetically Obese Rats for Studies in Diabetes, Heart Disease, and Complications of Obesity. NIH publication, Division of Research Services, Veterinary Resources Branch, National Institutes of Health, Bethesda, MD. 1988. p. 3-6.

10. Michaelis OE. New Models of Genetically Obese Rats for Studies in Diabetes, Heart Disease, and Complications of Obesity, Veterinary Resources Branch, Division of Research Services, NIH publication, Bethesda, MD, 1988.

11. Huang HJ, Young AA, Koda JE, et al. Hyperamylinemia, hyperinsulinemia, and insulin resistance in genetically obese $\mathrm{LA} / \mathrm{N}-\mathrm{cp}$ rats. Hypertension. 1992;19(1 Suppl):101-109.

12. Michaelis OE, Ellwood KC, Tulp OL, et al. Effect of feeding 1sucrose or starch diets on parameters of glucose tolerance in the $\mathrm{LA} / \mathrm{N}-$ corpulent rat. Nutr Res. 1986;6(2):95-99.

13. Tulp OL, Brown T. Effect of a fructan-chromium complex on glycemic responses of congenic obese LA/Ntul//-cp rats. J Nutr Health Food Eng. 2016;5(2):594-598

14. Stock MJ, Rothwell N. Diet induced thermogenesis and energy flux through brown adipose tissue. In: Proceeding of the XII International Congress on Nutrition. In: Taylor TG, Jenkins NK, John Libbey, editors. London;1985. p. 321-325.

15. Tulp OL. The effects of experimental over nutrition on non-shivering thermogenesis and obesity in LA/N-cp rats, Comp Bloch Physiology. 1991;98A:567-574.
16. Danforth E Jr. The role of thyroid hormones and insulin in the regulation of energy metabolism. Am J Clin Nutr. 1983;38:1006-1017.

17. Tulp OL, Sheilds SJ. Thermogenesis in cafeteria-fed LA/N-cp (Corpulent) rats. Nutr Res. 1984:4:325-332.

18. Marette A, Tulp OL, Bukowiecki LJ. Mechanism linking insulin resistance to defective thermogenesis in brown adipose tissue of obese diabetic SHR/N -cp rats. Int J Obese. 1991;15:823-831.

19. Bukowiecki LJ, Deshaies Y, Collet AJ, et al. Major thermogenic defect associated with insulin resistance in brown adipose tissue of obesediabetic SHR/N -cp rats. Am J Physiol.1991;E204-213.

20. Atgie C, Marette A, Desaultels M, et al. Specific decrease in mitochondrial thermogenic capacity in brown adipose tissue of obese $\mathrm{SHR} / \mathrm{N}-\mathrm{cp}$ rats. Am J Physio. 1993;265 C:1674-1680.

21. Himms-Hagen J. Thermogenesis in BAT as an energy buffer. NEJM. 1983;311:1150-1156.

22. Tulp OL, Root D, Frink R. The effect of anti-hypertensive drug treatment on brown adipocyte diameter and locule distribution in rats. Comp Biochem Physiol. 1984;79C(2):317-320.

23. Sclafani A, Xenakis S. Sucrose and polysaccharide induced obesity in the rat. Physiol Behav. 1984;32:169-174.

24. Sclafani A. Dietary carbohydrate preferences in the rat. Sattelite American Symposium Annual Neuroscience Meeting San Antonio San Antonio TX. 1985.

25. Marchington D, Rothwell NJ, Stoc MJ, et al. Energy balance diet-induced thermogenesis and brown adipose tissue in obese (fa/fa) Zucker rats after adrenalectomy. J Nutr. 1983;113:1395-140.

26. Young RA, Tulp OL, Horton ES. Thyroid and growth responses of young Zucker obese and lean rats to a low protein-high carbohydrate diet. $J$ Nutr. 1980;110:1421-1431.

27. SMJeyakumar, M Raja Gopal Reddy, C Garlapati, et al. Diabetogenic diet-induced insulin resistance associates with lipid droplet proteins and adipose tissue secretome, but not with sexual dimorphic adipose tissue fat accumulation in Wistar rats. Biochemistry and Biophysics Reports. 2020;24:100831.

28. Sclafani A, Xenakis S. Sucrose and polysaccharide induced obesity in the rat. Physiol Behav. 1984;32:169-174.

29. Sclafani A. Dietary carbohydrate preferences in the rat. Satellite American Symposium Annual Neuroscience Meeting San Antonio San Antonio TX.1985.

30. Tulp OL, Carlin C. Carbohydrate overnutrition induces increased adiposity in rats. Obesity Update. 1993;1-2.

31. Tulp OL, Einstein GP. Thermogenesis, aging and obesity in the LA/Ntul//cp (corpulent) rat. Adv Obes Weight Manag Control. 2021;11(2):37-43.

32. Tulp OL. Sympathetic and Thyroidal Components of Diet-Induced Facultative Thermogenesis in the LA-Corpulent Rat. Brain Res. Bull. $1985 ; 348$.

33. McCann UD, Shaw EA, Kaplan MM. Iodothyronine deiodination reaction -types. :effects of age,thyroid status,and glucocorticoid treatment. Endocrinology. 1984;114:1513-15213.

34. Trayhurn P. Origins and early development of the concept that brown adipose tissue thermogenesis is linked to energy balance and obesity. Biochimie. 2017;134:62-70.

35. Vedula U, Schnitzer-Polokoff R, Tulp OL. The effect of acarbose on the food intake, weight gain, and adiposity of $\mathrm{LA} / \mathrm{N}-\mathrm{cp}$ rats. Comp. Biochem. Physiol A Comp Physiol. 1991;100:477-482.

36. Ott L. Multiple comparisons. In:An Introduction to Statistical Methods and Data Analysis. 3rd ed. PWS-Kent, Boston MA. 1988;437-466. 
37. Tatleman HM, Tyzbir RS, Tulp OL. Effects of overfeeding on BAT and liver mitochondrial metabolism and shuttle activity in adult rats. Fed Proc. 1981;40:871.

38. Galton DA, Bray G. Metabolism of $\alpha$-Glycerol Phosphate in Human Adipose Tissue in Obesity. The Journal of Clinical Endocrinology \& Metabolism. 1967;27(11):1573-1580.

39. Tulp OL, Paz Nava M, Black DE, et al. Genetic determinants of T3 receptor affinity and thermogenesis in LA/N-cp rats" Proceedings, $X V$ International Congress of Nutrition Adelaide. International Union of Nutritional Sciences. 1993; book 1:481.

40. Kleiber M. The Fire of Life:An Introduction to Animal Energetics. Wiley publishers, New York NY USA; 1961
41. Wang ZM, Zhang J, Ying Z, et al. Organ-Tissue Level Model of Resting Energy Expenditure Across Mammals:New Insights into Kleiber's Law. Int Scholarly Research Network. IBSN Zoology. 2012;Art ID 673050 .

42. Kahle EB, Dadgari JM, Dudley GA, et al. Adaptive Response of Enzymes of Carbohydrate and Lipid Metabolism to Exercise, in New Models of Genetically Obese Rats for Studies in Diabetes, Heart Disease, and Complications of Obesity. Veterinary Resources Branch, Division of Research Services, NIH publication, Bethesda, MD. 1988;143-148.

43. Sims EAH. Experimental obesity, dietary-induced thermogenesis, and their clinical implications. Clinics in Endocrinology and Metabolism. 1976;5(2):377-395. 